Michael Fehler, EES-4

Rafael Benites (New Zealand Institute of Geological and Nuclear Sciences)

Peter Roberts, EES-4

Ru-Shan Wu (University of California, Santa Cruz)

Xiao-Bi Xie, EES-4

Submitted to:

DOE Office of Scientific and Technical Information (OSTI)

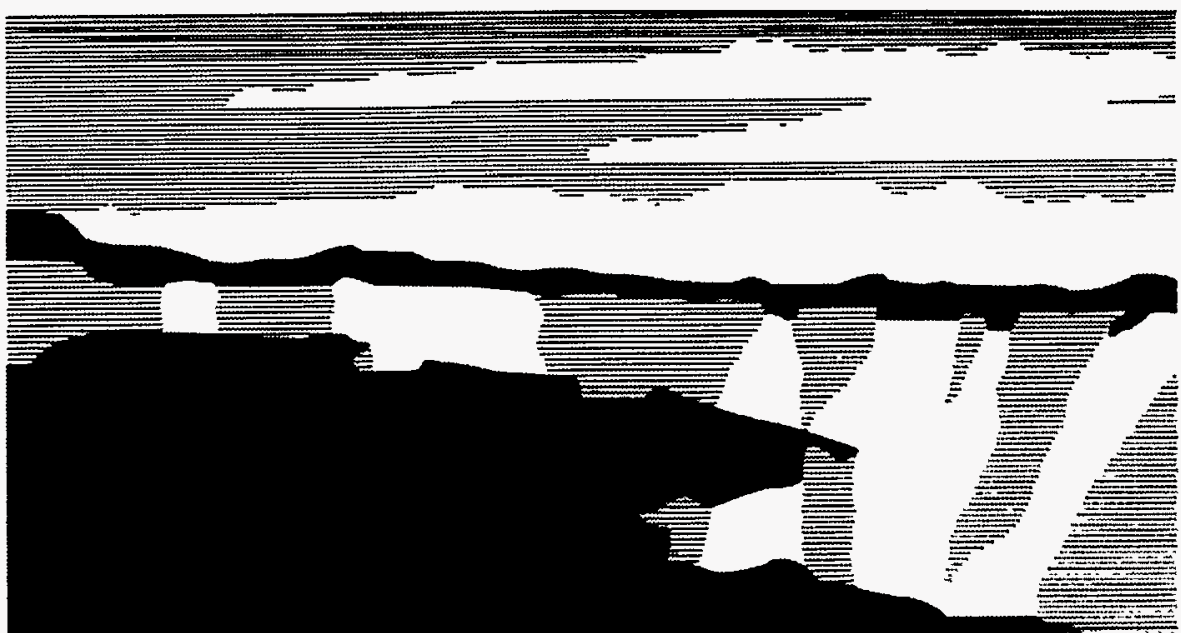

Los Alamos Natlonal Laboratory, an affirmative actionequal opportunity employer, is operated by the University of Califomia for the U.S. Department of Energy under contract W-7405-ENG-36. By acceptance of this article, the publisher recognizes that the U.S. Government retains a nonexclusive, royaltyfree license to publish or reproduce the published form of this contribution, or to allow others to do so, for U.S. Govemment purposes. The Los Alamos National Laboratory requests that the publisher identify this article as work performed under the auspices of the U.S._Department. of Energy. 


\title{
Stochastic Imaging of Oil and Gas Reservoirs
}

Michael Fehler*, Rafael Benites (New Zealand Institute of Geological and Nuclear Sciences), Peter Roberts, Ru-Shan Wu (U. of California, Santa Cruz), and Xiao-Bi Xie

\begin{abstract}
This is the final report of a three-year, Laboratory-Directed Research and Development (LDRD) project at the Los Alamos National Laboratory (LANL). In this project, we have been developing and testing methods for modelling and inversion of wave propagation through complex media. We have developed methods for modelling wave propagation in media that is characterized by both deterministic and stochastic components. Four methods have been successfully developed and tested to date: (1) an efficient but approximate method to model wave propagation in complex media, (2) an exact method for modelling wave propagation in complex media using a boundary integral approach, (3) a method for determining properties of a one-dimensional (1D) media composed of a sequence of thin layers each with random thickness and velocity, and (4) modelling of diffraction and resonance due to a low-velocity body. Our most recent efforts focused on extending the approximate method for modeling wave propagation in complex media to include the effects of singly reflected waves, which is essential for modeling seismic data collected by the petroleum industry. In addition, an effort has been made to quantify the limitations of the method.
\end{abstract}

\section{Background and Research Objectives}

In oil and gas exploration, there is increased interest in obtaining improved threedimensional (3D)images of selected regions of the earth's subsurface to locate potential oil and gas reservoirs. Methods such as ray-theory-based tomography, Born inversion, and timereverse migration have seen considerable application in all areas of earth imaging. Current methods used by the petroleum industry for characterizing subsurface structure are based on the acoustic wave equation. To obtain more detailed information about the earth's subsurface, better methods for modeling and processing seismic data using the elastic wave equation are needed.

\footnotetext{
*Principal investigator, e-mail: fehler@lanl.gov
} 
Migration is the name of the process by which surface seismic data collected by the petroleum industry is transformed into an image of the earth's subsurface. Currently, employed methods are difficult to apply to three-dimensional data because the computational resources required to perform the migration process are too large, or because the approximations made to reduce the computation cost adversely affect the quality of the image obtained. Currently, popular methods include time-reverse migration and Kirchhoff methods, but there are severe limitations for both of these methods that limit the quality of the subsurface image obtained, particularly in areas of complex subsurface structure. New methods to perform migration of seismic data for heterogeneous structures are thus required if reliable three-dimensional images are to be obtained in complex media.

We have been investigating alternative methods to image the earth using seismic data. In one method, we describe the spatial variations in material properties of the earth in a statistical sense and attempt to find the statistical parameters to characterize the variations. We thus obtain an image of the spatial distribution of medium heterogeneity that can be used to characterize in situ fracture systems, which are potential petroleum reservoirs. As part of this work, a method to rapidly calculate multiply forward-scattered and singly back-scattered energy in complex media has been developed. Not only does this method provide a fast way to model seismic wave propagation in complex media, it shows promise as an improved method to conduct elastic depth migration of seismic data.

Our goal was to develop and test new methods for seismic imaging and modeling of wave propagation in realistic complex elastic media. We focused on methods that allow us to more fully characterize media in both a deterministic and stochastic sense. This approach provides more detailed information about the earth's subsurface in regions of interest for oil and gas exploration and for basic science investigations.

\section{Importance to LANL's Science and Technology Base and National R\&D Needs}

The most direct application of our methods is in the petroleum exploration industry, where our methods are gaining a great deal of interest. The frontiers for petroleum exploration in the US occur in regions where drilling is expensive and that are difficult to image. Locations of exploration holes are chosen based on seismic images of the earth's subsurface. Imaging of media with smoothly varying properties is a well-studied problem and many successful approaches exist to do such imaging. When media become more complex, either due to the scale of fluctuation of media parameters or due to the range of media parameters, imaging 
becomes more difficult. Improved methods of imaging are required to make petroleum exploration in these areas economical.

An example of such a situation is in the US Gulf Coast, where many salt structures exist. These salt structures act as traps for petroleum reservoirs. Obtaining reliable images beneath large salt structures is important for identifying locations of sub-salt reservoirs. Obtaining such images is difficult in practice because of the large velocity contrasts between the salt and surrounding strata and because of the large range of orientations of interfaces associated with these contrasts. Similar problems with complexity occur for shallow environmental remediation sites, where near-surface heterogeneity can obscure images of the deeper structure. Improved understanding of wave propagation in such complex media is an essential first step to obtaining better images. Improved imaging techniques need to be developed and demonstrated to allow better information to be obtained about the subsurface of such complex regions.

This LDRD project provided the scientific underpinnings for a follow-on project funded by the Advanced Computational Technology Initiative in collaboration with several US petroleum companies.

\section{Scientific Approach and Results}

Our approach has been to investigate wave propagation in complex media using a suite of methods, from forward modeling of acoustic and elastic wave propagation in heterogeneous media, to investigating some of the difficulties encountered when doing conventional traveltime tomography. We have also investigated the range of applicability of models of wave propagation in random media. During the final year of the project, we focused on modeling seismic reflection data similar to that collected for petroleum exploration. These data are considered to be multiply forward scattered and singly back scattered. The modeling method involved the use of the De Wolf approximation in the complex phase screen method. Progress has been made on many fronts as described below.

\section{a. Seismic wave propagation and diffraction by large-scale complex}

visco-elastic structure. An efficient wave propagation algorithm for complex visco-elastic media is essential for improving 3D imaging in complex geological environments, such as oilgas or geothermal reservoirs. Based on the newly developed elastic complex-screen method of wide-angle one-way elastic wave propagation [1], we further improved the method and enhanced its range of application by introducing anelasticity (absorption) and oblique incidence into the method and programs. The elastic complex-screen (ECS) method uses dual domain implementation [transforming between the space and wave-number domains by Fast Fourier 
Transform (FFT)] and is much faster than a full-wave finite-difference program. For a moderate size $3 \mathrm{D}$ problem, the time saving is more than 2-3 orders of magnitude. Because it needs to store the medium parameters on only one grid-plane for each step, the tremendous computer memory saving makes it capable of handling large 3D problems that are prohibitive to other methods.

\section{b. The influence of small-scale random heterogeneities on seismic wave} propagation and imaging. Small-scale random heterogeneities can produce long scattered-wave trains known as codas, reduce the spatial and frequency coherence of the major arrivals and cause complicated effects due to apparent attenuation and dispersion. Therefore, it is important to study the influence on forward modeling and imaging of seismic waves due to the presence of such random heterogeneities, in addition to large scale structures. Using our ECS program, we can study numerically an important part of this random scattering: the forward scattering by random heterogeneities.

We have also been working to model the complete wavefield using a boundary integral approach $[2,3]$. While this modeling technique is more time consuming and can only model deterministic structure, we have been investigating complex structures with the program to obtain more information about where structural complexity can account for observed phenomena [4].

\section{c. Numerical experiments on separation of scattering and absorption in} randomly layered sediments. Oil-gas reservoirs are usually situated either beneath or inside a sedimentary layer that is composed of many thin-layers with random thickness and velocity. Therefore, it is important to understand the response of the sedimentary layer, modeled as a $1 \mathrm{D}$ random slab, to wave propagation and its influence on imaging. The randomlayered slab can affect both the primary and coda waves. The apparent attenuation caused by a random slab is an important factor for Q-tomography. Work has been done to extract the essential parameters of the random slab from the observed wave field characteristics. However, due to the combination of scattering and absorption during wave propagation, there has been no effective method to separate these two phenomena and estimate the corresponding parameters. We adopted a new approach, the energy transfer theory, which has been introduced into seismology for 3D problems, but has not been applied to the random-layered slab problem. Recently, the corresponding 1D theory was derived by one of the authors [5]. The theory has been tested on simulated data to outline the range over which the theory can be used to estimate media parameters from field data. 


\section{d. Diffraction and resonance scattering of a low-velocity body in elastic}

media and the implications to Q-tomography. Ray-tomography, including travel-time tomography and Q-tomography, has been widely used for cross-hole imaging of targets such as reservoirs. However, the validity and application range of such ray-approximated techniques has never been investigated. Especially for a low-V low-Q body, the limitations and difficulties of ray-tomography become an important issue. In order to attack this problem, we have studied the exact solutions of a visco-elastic cylinder by eigenfunction expansion method $[6,7]$ and the corresponding influence of wave diffraction and resonance scattering to ray-tomography.

\section{e. Seismic modeling using the multiple-forescattering single-} backscattering approach. During discussions with representatives from the petroleum industry while preparing a proposal for DOE funding to continue this work, we found that industry was extremely interested in the phase-screens approaches for modeling of seismic data. Modeling is the reverse of migration but has more stringent requirements than does migration. When discontinuities inside a medium are not very sharp or parameter perturbations of heterogeneities are not very strong, reverberations between heterogeneities or resonance scattering may be neglected. However, the accumulated effect of forward scattering usually can not be neglected. In such cases, the Born approximation is not valid but the one-return approximation (De Wolf approximation) can be applied. After renormalization of the multiple scattering series, De Wolf derived a MFSB (multiple-forescattering single-backscattering) approximation. Based on the De Wolf approximation, we developed two versions of dualdomain fast algorithms of synthetic seismograms:

- A wide-angle dual-domain thin-slab algorithm has been developed. Numerical tests for acoustic media have proved its validity and wide-angle capacity. The comparison with theoretical reflection coefficients for a flat interface showed that good agreements can go beyond 70 degrees of incident angles.

- Efficient screen approximation algorithms have been developed for acoustic and elastic back-scattered field calculations. Good agreement with finite-difference calculations has been obtained in numerical tests. 


\section{References}

[1.] Wu, R. S., "Wide-Angle Elastic Wave One-Way Propagation in Heterogeneous Media and an Elastic Wave Complex-Screen Method," J. Geophys. Res., 99, 751-766 (1994).

[2.] Benites, R., J. Haines, P. Roberts, and M. Fehler, "Boundary Integral Calculations of 2D Models Containing Inclusions," Abstracts of the International Association of Seismology and Physics of the Earth's Interior held in Wellington, New Zealand (1994).

[3.] Benites, R. and J. Haines, "Effects of Near-Surface Geology on Strong Ground Motion: A Review of the Advances in Numerical Modelling in New Zealand," Abstracts of the International Association of Seismology and Physics of the Earth's Interior held in Wellington, New Zealand (1994).

[4.] Roberts, P. M., R. Benites, M. Fehler, C. Thurber, L. Steck, W. Lutter, D. Stafford, and T. Zeichrt, "Teleseismic Waveform Anomalies Observed During the Passive 1993 Jemez Tomography Experiment (abstract)", Seismological Research Letters (1994).

[5.] Wu, R.S., "Spatial and Temporal Energy Distribution of Multiple-Scattered Waves in 1D Random Media and the Separation of Scattering from Absorption, I. Theory," submitted to Geophysics ( 1994).

[6.] Liu, Y.B. and R.S. Wu, "Scattering and Attenuation of Elastic Wave by a Visco-Elastic Cylinder," submitted to Expanded Abstracts of the Technical Program, SEG 63th Annual Meeting (1994).

[7.] Liu, Y.B. and R.S. Wu, "Scattering and Attenuation of Elastic Wave by a Visco-Elastic Cylinder, I. P-Wave Incidence, preprint ( 1994). 